\section{Exaggerated serum levels of alpha-fetoprotein in focal nodular hyperplasia of the liver}

\author{
Lorenzo Cirri, ${ }^{1,2}$ Farouk Dahmash, ${ }^{1}$ \\ Christian Marin, ${ }^{1}$ Wolfram Grüning, ${ }^{2}$ \\ Ahmet H. Elmaagacli ${ }^{1}$ \\ ${ }^{1}$ Hematology and Oncology, and \\ ${ }^{2}$ Respiratory Medicine, Schwerin Medical \\ Center, Germany
}

\section{Abstract}

Alpha-fetoprotein (AFP) is a tumor marker routinely used for the diagnosis and follow-up of malignant neoplasms. We report a case of extremely high serum levels of AFP associated with benign lesions of the liver and of the lungs with a spontaneous and gradual drop to normal values in the 9-month follow-up without any form of treatment. As a result a high serum level of AFP does not necessarily indicate a malignant tumor and every patient presenting with such finding must be thoroughly examined before making a definitive diagnosis.

\section{Introduction}

Alpha-fetoprotein (AFP) is a major plasma protein physiologically produced by the yolk sac and the liver during fetal development. The normal AFP serum level in adult men and nonpregnant women should be under $10 \mathrm{ug} / \mathrm{L}$. An increase in AFP level is associated with active regeneration of hepatocytes, as in the presence of a tumor. ${ }^{1}$ AFP-secreting tumors include hepatocellular carcinoma (HCC) and other mainly gastrointestinal or gem cell malignant neoplasms. In patients affected by these tumors, serum levels of AFP often correlate with tumor size and surgical resection or effective chemotherapy is usually associated with its drop. AFP is therefore a useful marker to help making the correct diagnosis as well as assessing response to treatment.

The association between AFP and benign tumors is very rare and only a few cases have been described in the literature. We present a patient with focal nodular hyperplasia (FNH) of the liver and nonspecific reactive pulmonary nodules whose AFP serum level at presentation exceeded 6000 times the normal value. The exaggerated AFP-value spontaneously and gradually dropped to normal values in the 9month follow-up.

\section{Case Report}

An 82-year-old male patient was referred by his general practitioner to the Department of Hematology and Oncology of Schwerin Medical Center, a tertiary hospital in northern Germany, due to unexplained weight loss of about $4 \mathrm{~kg}$ over the previous 3 months as well as abnormal findings on a routine chest $\mathrm{x}$-ray.

The patient had a history of excessive alcohol consumption and smoking until about 20 years before, apart from that no history of any chronic disease. No association with any occupational pathogen and no exposure to Mycobacterium tuberculosis could be identified. The physical examination did not reveal any significant abnormality, apart from a tender and slightly enlarged liver of about $3 \mathrm{~cm}$ under the costal arch. Laboratory investigations revealed a mild normochromic normocytic anemia (7.8 mmol/L, normal range 8.6-12.0) and a mild increase of bilirubin $(29 \mathrm{umol} / \mathrm{L}$, normal range $<17$ ). All other routine parameters and liver function tests were normal. Serologically there were no sign of present or past hepatitis or HIV infection. The Quantiferon-Gold ${ }^{\mathrm{TM}}$ test was negative. Among all tested tumor markers the only significant increase was registered for Alpha-fetoprotein at $60.187 \mathrm{ug} / \mathrm{L}($ normal $<10)$ and therefore a malignant tumor was suspected.

In order to secure the diagnosis we performed a computed tomography (CT) scan of chest, neck and abdomen. Here a big lesion of the right liver lobe measuring about $10 \times 9.5 \mathrm{~cm}$ with signs of early stage liver damage as well as multiple bilateral pulmonary nodules of maximum $8 \times 6 \times 5 \mathrm{~mm}$ was described. Both pulmonary and liver structures underwent a CTguided biopsy. Histologically there was no evidence of malignant cells, fibrosis or inflammation in the biopsy material from the lung, while the biopsy material from the liver revealed FNH (Figure 1). Abdominal magnetic resonance imaging, gastroscopy, colonoscopy and bronchoscopy with broncho-alveolar lavage, did not add any relevant information (Figure 2). In absence of malignancy, the clinically stable patient was diagnosed with FNH of the liver as well as nonspecific reactive pulmonary nodules and was released from our hospital without any treatment, being offered a short time follow-up. On discharge (day 15) a first substantial AFP drop was registered (now at $13.371 \mathrm{ug} / \mathrm{L}$, a $77 \%$-decrease compared to the previous value).

The first follow-up was performed after two weeks (day 30) and a CT scan was repeated. The patient was feeling good and had normal appetite. At this time the lung nodules appeared to be decreased both in quantity and in size and the FNH of the liver also appeared
Correspondence: Lorenzo Cirri, Departments of Hematology and Oncology; and Respiratory Medicine, Schwerin Medical Center, 19055,

Schwerin, Germany.

E-mail: cirri.lorenzo@gmail.com

Key words: Tumor markers; Liver; FNH.

Contributions: the authors contributed equally.

Conflict of interest: the authors declare no potential conflict of interest.

Received for publication: 6 January 2016. Accepted for publication: 12 August 2016.

This work is licensed under a Creative Commons Attribution NonCommercial 4.0 License (CC BYNC 4.0).

(C) Copyright L. Cirri et al., 2016

Licensee PAGEPress, Italy

Gastroenterology Insights 2016; 7:6399

doi:10.4081/gi.2016.6399

smaller in size, now at about $6.5 \times 6.5 \mathrm{~cm}$. Moreover AFP had remarkably decreased at $5.293 \mathrm{ug} / \mathrm{L}$ (60\% decrease compared to the previous value).

In the second follow-up (day 60) a further decrease in size of both liver and lung nodules (now almost disappeared) was described. AFP was now at $3.632 \mathrm{ug} / \mathrm{L}$ (a further decrease of about $22 \%$ ) and our asymptomatic patient was gaining weight (Figure 3 ).

We performed other three follow-ups at day 90,120 and 270 , where a further decrease of AFP was described (83.6, 94.4 and 90.9\% drop compared the previous value respectively). At day 270 (9 months after first presentation) AFP was normal (3 ug/L) and radiological abnormalities had completely disappeared.

\section{Discussion and Conclusions}

The reported case describes a rare association between very high levels of AFP and nonmalignant lesions of liver and lungs. Reviewing the literature we only found a paper that reported elevated levels of AFP in a patient with FNH. ${ }^{2}$ The value described was at around $114 \mathrm{ug} / \mathrm{L}$ much lower than the one we report. Furthermore a spontaneous drop in AFP levels without any form of treatment has not been described yet. We conclude that highly increased serum levels of AFP do not always indicate the presence of HCC or other malignancies, but may be found also in non-malignant liver lesions such as FNH. AFP serum levels should therefore be re-checked at regular 


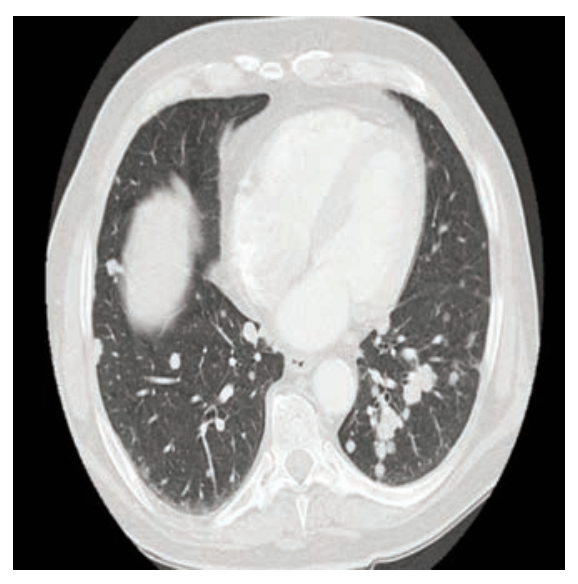

Figure 1. Multiple bilateral lung nodules on computed tomography scan at presentation. Histology excluded the presence of malignant cells.

intervals, as a spontaneous drop may be possible even in absence of therapy.

An association between FNH and nonspecific reactive lung nodules may be possible. Further studies are needed to prove this association.

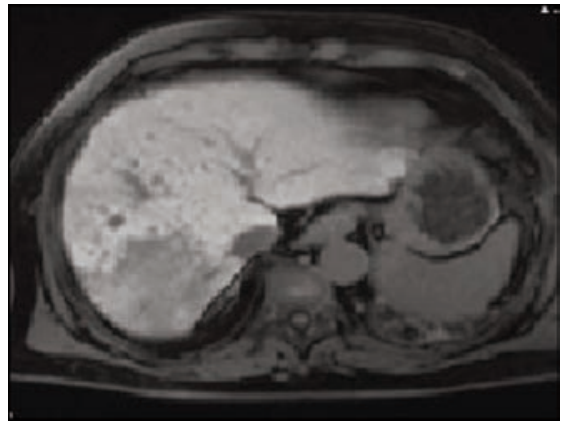

Figure 2. Focal nodular hyperplasia of the liver on magnetic resonance imaging at presentation.

\section{References}

1. Kakisaka K, Kataoka K, Onodera M, et al. Alpha-fetoprotein: a biomarker for the recruitment of progenitor cells in the liver in patients with acute liver injury or fail-

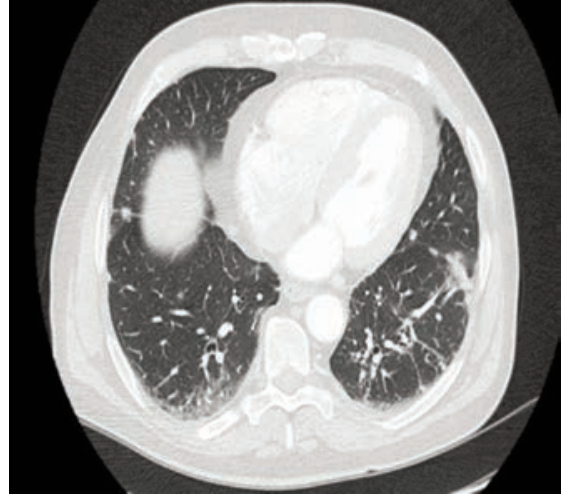

Figure 3. Spontaneous regression of lung nodules on second follow-up (day 60). No therapy had been performed.

ure. Hepatol Res 2015;45:E12-20.

2. Wadad M, Farges 0, Bedossa P, et al. High serum level of alpha-fetoprotein in focal nodular hyperplasia of the liver. Pathol Int 2011;61:536-8. 